with a higher total disease activity score (SLEDAI $\geq 6$ ) (OR 9.9, 95\% Cl 2.1 to $49.9, p=0.0056$ ), neither of the single SLEDAI items and organ manifestations including musculoskeletal manifestations was associated with physical inactivity. Interestingly, the study could not detect any statistical difference in organ manifestations and SLEDAI scores between patients with moderate and high PA. According to the patient's report, the main SLE-related reasons "not to be physically active" for all three groups were "lupus flare" (35.6\%), "fatigue" $(26.9 \%)$ as well as "joint complaints" $(15.7 \%)$. The main general barriers for PA were "comorbidity" (35,6\%) and "lack of motivation" (26,9\%). Furthermore, the subjective impact of "bad weather conditions" on physical activity was significantly greater in physically inactive patients compared with the two other groups (the patient's report: $6.58 \pm 2.3$ vs. $3.49 \pm 0.7, p<0.03$ ).

Conclusions: The main reason for the patients not to be physically active was fatigue and pain. The study also indicates that not only somatic symptoms could decrease the levels of PA in SLE patients. Further research on psychological factors is needed. The study underlines the need for management strategies that specifically target physical activity as a part of an overall SLE management program.

Disclosure of Interest: None declared

DOI: 10.1136/annrheumdis-2017-eular.6024

\section{THU0278 COMPARISON OF THE 2016 ACR/EULAR AND THE 2002 AECG CLASSIFICATION CRITERIA IN A COHORT OF PATIENTS WITH SUSPECTED PRIMARY SJÖGREN'S SYNDROME}

M. Le Goff ${ }^{1}$, D. Cornec ${ }^{2}$, S. Jousse-Joulin ${ }^{2}$, D. Guellec ${ }^{2}$, S. Costa ${ }^{2}$,

T. Marhadour ${ }^{2}$, R. Le Berre ${ }^{2}$, S. Genestet ${ }^{2}$, B. Cochener ${ }^{2}$,

S. Boisrame-Gastrin ${ }^{2}$, Y. Renaudineau ${ }^{2}$, J.-O. Pers ${ }^{2}$, A. Saraux ${ }^{2}$,

V. Devauchelle-Pensec ${ }^{2} .{ }^{1}$ Rheumatology; ${ }^{2}$ CHRU Brest, Brest, France

Background: New consensual classification criteria for primary Sjögren's syndrome (pSS) have been recently developed and endorsed by ACR and EULAR. They differ substantially from previously used AECG criteria in that they consider systemic involvement (defined as ESSDAI score $\geq 1$ ) as well as sicca symptoms as entry criteria before applying a weighted score. Evaluation of the concordance and differences between the two sets of criteria in independent patient populations is mandatory to establish how future clinical studies using the new criteria will be comparable to previously published studies. Major salivary gland ultrasonography (SGUS) has demonstrated promising diagnostic performance in previous studies, but was not included in these new classification criteria.

Methods: This cross-sectional study was conducted in the monocentric Brittany cohort (DIApSS cohort) of patients with suspected pSS (sicca symptoms, parotidomegaly or extraglandular manifestations suggestive of pSS). All patients had standardized clinical examination, basic biology, immunological tests and minor labial salivary gland biopsy. SGUS in mode B was performed by the same experienced operator, who was blinded to the diagnosis. Agreement between the two sets of criteria was assessed using Cohen's $\kappa$ coefficient and the characteristics of discordant patients were detailed.

Results: 290 patients were prospectively included between 2006 and 2016 . Mean age was $55.6 \pm 13.2$ years, $92.1 \%$ were female and mean duration of the symptoms $6.0 \pm 6.6$ years. More patients fulfilled ACR/EULAR criteria $(n=125$, 43.1\%) than AECG criteria ( $n=114,39.3 \%)$. 114 patients (39.3\%) fulfilled both criteria, 11 (3.8\%) fulfilled ACR/EULAR only, O AECG only and 165 (56.9\%) none of the criteria. Concordance between both criteria was good (kappa $=0.9$ ). Compared to patients fulfilling both criteria, patients fulfilling ACR/EULAR but not AECG criteria $(n=11)$ had similar age, similar symptom duration, but less frequent sicca symptoms (eye dryness $18.2 \%$ versus $96.5 \%$; mouth dryness $54.5 \%$ versus $97.4 \%, p<0.01$ for both), and less frequent salivary gland dysfunction (salivary flow $\leq 0.1 \mathrm{ml} / \mathrm{min}$ : $20 \%$ versus $70.9 \%, p<0.01$ ). They had characteristic features of pSS, with frequent systemic involvement at diagnosis $(90.9 \%)$, positive salivary gland biopsy $(90.9 \%)$, abnormal SGUS $(44.4 \%)$ and presence of anti-SSA/SSB autoantibodies (45.4\%). $46.5 \%$ of them had a diagnosis of pSS according to the physician. Among patients negative for the two sets of criteria, $12 \%$ had an abnormal SGUS and $10.3 \%$ received a clinical diagnosis of pSS based on physician opinion.

Conclusions: Agreement between AECG criteria and new ACR/EULAR criteria is good suggesting that they select quite similar patients. ACR/EULAR criteria display a slightly higher sensitivity and are able to detect more patients with systemic involvement, but some of these patients did not have pSS according to the physician diagnosis. As previously demonstrated for AECG criteria, SGUS inclusion into ACR/EULAR criteria may further enhance their sensitivity.

Disclosure of Interest: None declared

DOI: 10.1136/annrheumdis-2017-eular.5446

\section{THU0279 ARE THE PULMONARY INVOLVEMENT IN SYSTEMIC LUPUS ERYTHEMATOSUS ASSOCIATED WITH A HIGHER PREVALENCE OF COMORBIDITIES?}

M. Cebanu ${ }^{1}$, V. Salaru ${ }^{2}$, V. Sadovici ${ }^{3}$, M. Pasali ${ }^{3}$, N. Loghin-Oprea ${ }^{3}$, M. Mazur ${ }^{3}$, L. Mazur ${ }^{4}$, G. Ciobanu ${ }^{1} .{ }^{1}$ Emergency Medicine; ${ }^{2}$ Family Medicine; ${ }^{3}$ Rheumatology; ${ }^{4}$ Cardiology, State University of Medicine and Pharmacy "Nicolae Testemitanu", Chisinau, Moldova, Republic of

Background: Patients with systemic lupus erythematosus have a very high burden of comorbidities. Identification and management of these comorbidities are critical for optimal medical care to this population.

Objectives: To assess the prevalence of comorbidities in SLE patients with pulmonary involvement.

Methods: In a cross-sectional study, patients who fulfiled the SLICC (2012) classification criteria for SLE, were recruited from Rheumatology Departement. Data collection included demographics, disease duration, physician-rated indices of disease activity (by SLAM), damage (by SLICC/ACR DI) and Charlson comorbidity Index. The pulmonary involvement was assessed by chest $\mathrm{X}$-ray, EcoCG Doppler and pulmonary functional tests.

Results: The study included 106 patients (97 women, 9 males) with a mean age $( \pm S D)$ of $41,1 \pm 12,6$ yrs, mean disease duration of $90,3 \pm 87,3$ months. The disease activity by SLAM was $11 \pm 5,17$ points and mean SLICC/ACR DI 1,9 92,4 points $(66 \%$ of patients had at least 1 point). Pulmonary assessment revealed that $45(42,5 \%)$ patients had different types of pulmonary involvement due to lupus: pleuritis - 24 patients, pneumonitis -1 patient, pulmonary embolism -4 patients, interstitial lung disease -15 , shrinking lung syndrome -1 and pulmonary arterial hypertension - 9 patients. The most frequent comorbidities in study group were: arterial hypertension - in $57(53,7 \%)$ cases, from which $33(57,9 \%)$ patients had pulmonary involvement and $24(42,1 \%)$ without, obesity $\left(\mathrm{BMl}>30 \mathrm{~kg} / \mathrm{m}^{2}\right)$ had 29 $(27,4 \%)$ patients, from which $17(58,6 \%)$ with lung involvement and $12(41,4 \%)$ without, anemia $(\mathrm{Hb}<110 \mathrm{~g} / \mathrm{l})$ had $24(22,6 \%)$ patients, from them $14(58,3 \%)$ with lung disease and $10(41,7 \%)$ patients - without, heart failure (I-II NYHA) had 23 $(21,7 \%)$ patients, from them $20(86,9 \%)$ were with lung implication, thyroiditis had $22(20,8 \%)$ and $15(68,2 \%)$ of them were with pulmonary involvement, diabetes mellitus type II had only $6(5,7 \%)$ patients and half of them had lung disease. Assessing the impact of associated diseases through Charlson comorbidity index, we found that the score for patients diagnosed with damage to the respiratory system was twice as big vs. patients without respiratory impairment from SLE $(6,3 \pm 2,4$ vs. $3,4 \pm 1,4$ points). Also, Charlson comorbidity score $\geq 1$ was identified as a risk factor for lung involvement (OR 5,5294, 95\% Cl 2,367-12,91, p<0,01). Evaluation of disease activity by SLAM showed that patients with lung involvement have a higher disease activity vs. patients without $(13,9 \pm 6,0$ vs. $8,9 \pm 4,0, p<0,05)$. Conclusions: On the one hand, according to our results patients with SLE and pulmonary involvement have a higher prevalence of comorbidities comparative with patients without them. Hypertension was found to be the most common comorbidity and it was determined in $73,3 \%$ of patients with impaired respiratory system $(p<0,01)$. On the other hand, association of comorbidities (Charlson comorbidity score $\geq 1$ ) was identified as a risk factor for lung lesions.

References:

[1] Rees F., Doherty M., Grainge M. et al. The Burden of Comorbidity in Systemic Lupus Erythematosus. Rheumatology (2015), 54 (suppl_1): i166.

Disclosure of Interest: None declared

DOI: 10.1136/annrheumdis-2017-eular.6776

\section{THU0280 COMPARISON OF CLASSIFICATION CRITERIA FOR SJÖGREN'S SYNDROME FROM 2002 AND 2016 IN AN INCIDENT COHORT DIAGNOSED 2007 TO 2011 FROM STOCKHOLM COUNTY SWEDEN}

M. Kvarnström, M. Wahren-Herlenius. Dep. of Medicine, Unit of Experimental Rheumatology, Karolinska Institutet, Karolinska Uniververity Hospital, Stockholm, Sweden

Background: The current American-European Consensus Criteria (AECC) from 2002 has been the most widely used and applied all over the world. New classification criteria for Sjögren's syndrome was published in 2016, designed in a collaboration between ACR and EULAR. They are made up of a scoring system in which 4 points are required for classification. The weight/score is as follows:

- Labial salivary gland biopsy with focal lymphocytic sialadenitis and focus score of $>1 \mathrm{foci} / 4 \mathrm{~mm}^{2}-3$

- Anti-SSA/Ro positive -3

- Ocular Staining Score $>5$ (or van Bijsterveld score $>4$ ) in at least 1 eye -1

- Schirmer's test $\leq 5 \mathrm{~mm} / 5$ minutes in at least 1 eye -1

- Unstimulated whole saliva flow rate $\leq 0.1 \mathrm{ml} /$ minute -1 .

Objectives: Comparison of classification criteria for Sjögren's syndrome from 2002 and 2016 in a 5 year cohort of incident patients diagnosed 2007 to 2011 from Karolinska University Hospital, Stockholm County, Sweden.

We wanted to examine the consistency between the different classification criteria. Methods: We compared a cohort of all diagnosed patients with primary Sjögren's syndrome during the years 2007 to 2011 at the Dep. of Rheumatology at Karolinska University Hospital in Stockholm Sweden. Data on the item Ocular Staining Score $\geq 5$ was not available since it is not included in AECC. The cohort consisted of 199 patients all fulfilling the 2002 AECC. Another eight patients did 
not fulfill the criteria but were regarded as possible Sjögren's syndrome under development by the rheumatologist who investigated them.

Results: 196 of the 199 pSS patients also fulfilled the new criteria from 2016. The three patients who did not only had SSB autoantibodies and no positive biopsy. Of eight patients with suspicion of Sjögren's syndrome under development, three fulfilled the new criteria.

Conclusions: The classification criteria for Sjögren's syndrome from 2002 and 2016 are consistent. This study indicates a possibility that the new criteria may be more sensitive early in the disease development, as long as the classification is not based on autoantibody positivity against SSB.

Disclosure of Interest: None declared

DOI: 10.1136/annrheumdis-2017-eular.5377

\section{THU0281 LONG-TERM PROGNOSIS AND PREDICTINGFACTORS OF CHINESE PATIENTS WITH SYSTEMIC LUPUS ERYTHEMATOSUS: A MULTI-CENTER COHORT STUDY FROM CSTAR REGISTRY}

M. $\mathrm{Li}^{1}$, Z. Wang ${ }^{1}$, Y. Wang ${ }^{2}, \mathrm{X}$. Zeng ${ }^{1}$ on behalf of CSTAR. ${ }^{1}$ Peking Union Medical College Hospital; ${ }^{2}$ Chinese Academy of Medical Science, Beijing, China

Objectives: To investigate the long-term outcomes, both mortality and damage, and related prognostic factors of patients with systemic lupus erythematosus (SLE) in the CSTAR (Chinese SLE Treatment and Research group) registry cohort.

Methods: All of the patients were enrolled from April 2009 to February 2010. They were followed up at clinic and were telephone interviewed at the endpoint. Demographic data, clinical manifestations, activity (SLEDAI-2K), damage scores (SLLIC/Damage Index), and medications were collected. Data were censored at the last clinic visit or telephone interview. Survival rates were studied by Kaplan-Meier method, and COX proportional hazard model was adopted to perform the analysis of predicting factors for mortality.

Results: A total of 2104 patients were recruited at baseline, and 1494 patients were successfully followed up. The cumulative 1, 3 and 5-year survival ratesfrom diagnosis were $99.0 \%, 98.1 \%$ and $97.1 \%$. 78 patients died during follow-up, and the main death causes were infection (34.6\%), active disease (26.9\%), cardiovascular and cerebrovascular events $(6.41 \%)$ and malignancy $(5.13 \%)$. At entry, 247 patients presented with irreversible organ damage and it increased to 398 patients at the endpoint. The major accumulated organ damages were renal $(25.9 \%)$, musculoskeletal $(20.2 \%)$, neuropsychiatric $(12.4 \%)$, and pulmonary $(10.8 \%)$ damage. Cox regressionshowed that male, late onset age ( $>50 \mathrm{y})$, onset to diagnosis time $\geq 1$ year, previous organ damage, renal involvement, pulmonary arterial hypertension, neuropsychiatric involvement, serositis and the number of involved organ systems $\geq 3$ predict for higher mortality.

\begin{tabular}{llll}
\multicolumn{4}{l}{ Table 1: Independent predictors of mortality obtained by univariate analysis } \\
\hline Univariate analysis & $\mathrm{HR}$ & $95 \% \mathrm{Cl}$ & $p$ \\
\hline Age at onset $\geq 50$ years & 3.935 & $2.111-7.334$ & $<0.001$ \\
Onset to diagnosis $\geq 1$ year & 1.996 & $1.251-3.186$ & 0.004 \\
Gender & 2.082 & $1.119-3.873$ & 0.021 \\
Baseline organ damage* & 2.846 & $1.757-4.610$ & $<0.001$ \\
Renal involvement* & 2.434 & $1.425-4.156$ & 0.001 \\
Hematologic involvement* & 1.605 & $0.978-2.635$ & 0.061 \\
Interstitial lung disease* & 2.167 & $0.973-4.823$ & 0.058 \\
Pulmonary arterial hypertension* & 4.126 & $2.107-8.081$ & $<0.001$ \\
Neuropsychiatric involvement* & 2.290 & $1.169-4.486$ & 0.016 \\
Serositis* & 2.580 & $1.605-4.148$ & $<0.001$ \\
No. of involved organ systems (1) * & 1.793 & $0.719-4.466$ & 0.210 \\
No. of involved organ systems (2) * & 2.045 & $0.805-5.195$ & 0.133 \\
No. of involved organ systems ( $\geq 3)^{*}$ & 5.638 & $2.339-13.589$ & $<0.001$ \\
\hline
\end{tabular}

* Performed after controlling of gender, onset age and time from onset to diagnosis

Conclusions: Long-term survival rates have improved for Chinese SLE patients. Early diagnosis, preventing fromthe emerging systemic organ involvements and organ damagecould be the treating target for the management of SLE patients in China.

Disclosure of Interest: None declared

DOI: 10.1136/annrheumdis-2017-eular.5791

\section{THU0282 HOMOCYSTEINE: ANY ROLE IN PERIPHERAL VASCULAR DISEASE IN SYSTEMIC LUPUS ERYTHEMATOSUS (SLE) PATIENTS?}

N. Mohannad $^{1}$, M. Tayel ${ }^{2}$, M.H. Megallaa ${ }^{3} .{ }^{1}$ Internal Medicine, Rheumatology Unit, Alexandria University Hospitals, Alexandria University; ${ }^{2}$ Internal Medicine, Rheumatology Unit; ${ }^{3}$ Internal Medicine, Alexandria University, Alexandria, Egypt

Background: Many are the independent risk factors for premature atherosclerosis in general \& peripheral vascular disease (PVD) in particular in SLE patients. Plasma homocysteine (HCY) is a known risk factor for atherosclerosis. Atherosclerosis can lead to many cardiovascular diseases as myocardial infarction, stroke and claudication
Objectives: To compare the occurrence PVD of the lower extremity of SLE patients (pts) with age and sex matched controls and evaluate the role of HCY level in its occurrence

Methods: Body mass index (BMI), blood pressure, lipid profile, titers of autoantibodies [ANA, anticardiolipin antibodies ACL (IgM, IgG)], C3, C4, plasma HCY level were assessed, SLEDAI and (SLICC/ACRDI)were calculated. PVD evaluation was done by measuring Ankle Brachial Index (ABI) with values $<0.9$ considered diagnostic of PVD; in 60 SLE pts and 30 age-matched controls. Patients with previous hypertension, diabetes, other collagenic diseases \& smokers were excluded

Results: Eighty-eight percent of the pts were women. The mean age (SD) was 30.40 (11.46) years \& mean disease duration 3.61 (4.92)years. 50 pts were asymptomatic, 5 had mild \& 5 had moderate claudications. SLE pts had significant higher total cholesterol (TC), LDL than controls $224.1 \pm 57.8$ vs $181.1 \pm 41.1 \mathrm{mg} / \mathrm{dl}$ \& $162.7 \pm 57.0$ vs $119.5 \pm 13.4\left(<0.001^{*}\right)$ respectively, higher $\mathrm{HCY} 11.6 \pm 2.1$ \& $6.4 \pm 0.1 \mu \mathrm{mol} / \mathrm{L}\left(\mathrm{p}<0.001^{\star}\right)$ \& lower HDL $47.2 \pm 13.1 \& 52.6 \pm 3.7 \mathrm{mg} / \mathrm{dl}\left(\mathrm{p}=0.004^{\star}\right)$. Low $\mathrm{ABI}$ was found in $30 \%$ of SLE pts but none of the controls $\left(p=0.001^{*}\right)$ \& was correlated with higher HCY level $\left(p=0.005^{\star}\right)$, TC \& LDL $\left(p<0.001^{*}\right.$ \& $\left.p<0.001^{*}\right)$, but not TG $(p=0.748)$ or asymptomatic $p t s$, presence of mild or moderate claudications $(p=0.468,1.000,0.154)$, still its value negatively correlated with lupus anticoagulant (LA) $(p=0.002), A C L \operatorname{lgM}\left(p<0.001^{*}\right)$, the presence of lupus nephritis (LN) $\left(p<0.001^{*}\right)$ \& SLICC/ACRDI $\left(p=0.017^{*}\right)$ but not with disease duration (dd) $(p=0.535)$, Anti ds DNA ( $p=0.364)$, ACL IgG $(p=0.8940)$, C4 $(p=0.168)$ or SLEDAI $(p=0.074)$. No correlation was found between HCY level and pts' age, dd, age at diagnosis, BMI, Anti ds DNA, ACL IgG, C4 \& SLEDAI $(p=0.521,0.098,0.946,0.502,0.346,0.335,0.325,0.787)$. A positive correlation was found between HCY level and LA, ACL lgM, TC, LDL, the presence of LN \& SLICC/ACRDI with a p value of $0.025^{*},<0.001^{*},<0.001^{*}, 0.003^{*}, 0.001^{*}, 0.001^{*}$ \& negative one with $\mathrm{HDL} \mathrm{p}=0.023^{*}$
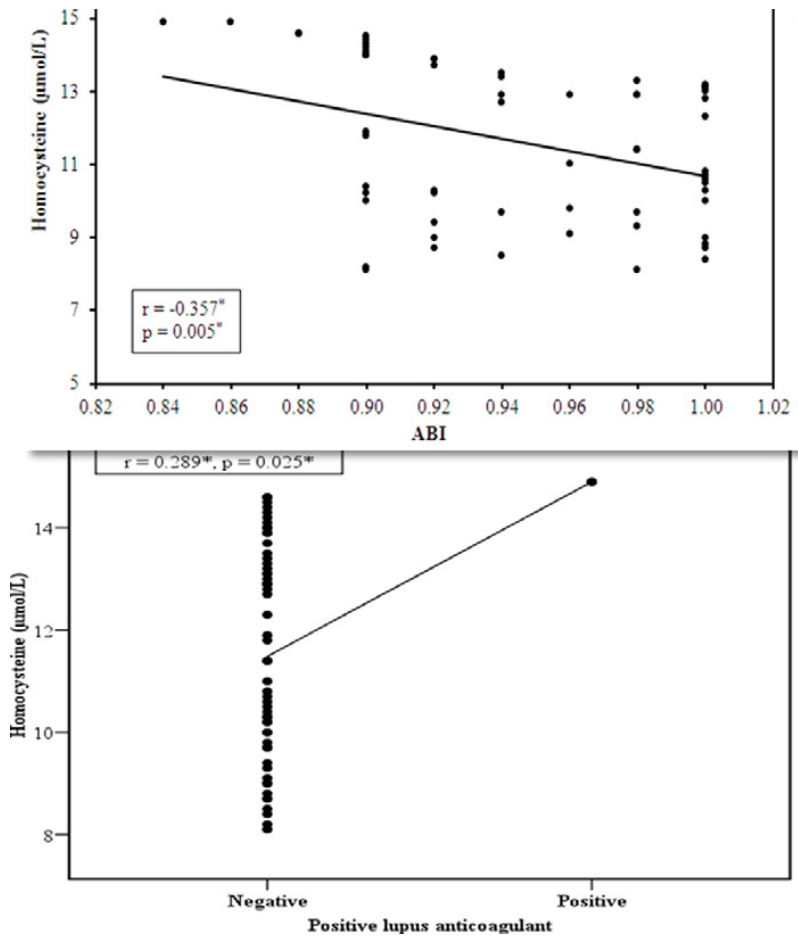

Conclusions: $83.3 \%$ of SLE pts were asymptomatic or had atypical symptoms of PVD still $30 \%$ of the patients had low $A B I$. ABI can be a more reliable, non-invasive test to assess PVD than the conventional methods of pulse palpation or history of claudication in SLE pts.Both traditional \& nontraditional risk factors of atherosclerosis are important but $\mathrm{HCY}$ can play a role, among other factors, as independent risk factor of PVD in SLE

References:

[1] Rayford R et al. PVD in SLE Patients. J Clin Rheum. 2013; 7:367-70.

Disclosure of Interest: None declared

DOI: 10.1136/annrheumdis-2017-eular.4615 